\title{
Incidencia del suicidio en las prisiones de Cataluña: análisis descriptivo y comparado
}

\author{
A Bedoya, PA Martínez-Carpio, V Humet, MJ Leal, N Lleopart
}

Servicios Médicos. Centre Penitenciari de Joves. La Roca del Vallès (Barcelona). Departament de Justícia. Generalitat de Catalunya.

\section{RESUMEN}

Introducción: La prevención del suicidio en prisión se impone entre los retos más importantes de la sanidad penitenciaria en los países desarrollados. El objetivo de este trabajo es analizar la incidencia del suicidio en las prisiones catalanas y recoger datos sobre algunas variables investigadas también en otras poblaciones de reclusos.

Material y métodos: Estudio retrospectivo de los casos de suicidio en los centros penitenciarios dependientes de la Generalitat de Catalunya entre 1990-2005 ( $\mathrm{n}=65)$. Estudio comparado de la incidencia con los países europeos a partir de datos publicados.

Resultados: La incidencia anual media del período es de 59/100.000, octuplicando la tasa correspondiente a la población general.

Conclusiones: El ingreso en prisión supone el acontecimiento desencadenante más importante. Otros resultados abren las puertas a nuevas investigaciones.

Palabras clave: Suicidio, Incidencia, Prisiones, España, Factores de Riesgo.

\section{INCIDENCE OF SUICIDE IN CATALAN PRISONS: A DESCRIPTIVE AND COMPARATIVE STUDY}

\section{ABSTRACT}

Introduction: The prevention of suicide in prison is one of the most important challenges facing prison health care services in developed countries. The aim of this study is to analyse the incidence of suicide in Catalan prisons and accumulate data about a number of variables that have also been studied in other prison populations.

Materials and methods: Retrospective study of suicide cases in prisons administrated by the Catalan Regional Government (Generalitat de Catalunya) between 1990 and $2005(\mathrm{n}=65)$. Comparative study of incidence with European countries using published data.

Results: The average annual incidence for the period is of 59/100.000, multiplying by eight the level corresponding to the general population.

Conclusions: Entry into prison is the most important event trigger for suicide. Other data open the way to new research.

Key words: Suicide, Incidence, Prisons, Spain, Risk Factors.

Texto recibido: octubre 2008

Texto aceptado: mayo 2009

\section{INTRODUCCIÓN}

El suicidio es un trágico problema sanitario, frecuentemente ocultado e ignorado, que conlleva un padecimiento importante del propio suicida y de sus familiares y personas próximas. Según la Organización Mundial de la Salud (OMS) en el año 2000 se suicidaron alrededor de un millón de personas, casi la mitad de las muertes violentas en todo el mundo. La tasa de suicidios asciende a 16/100.000 habitantes, 
siendo más frecuente en Europa y Asia. En Europa esta tasa es de 20, en España 8,3 y en Cataluña 7,9. Ocurren más muertes por suicidio que la suma de homicidios y guerras, y a nivel global la mortalidad por suicidio es equiparable a la que ocurre por accidentes de tráfico ${ }^{1-3}$.

El factor de encarcelamiento multiplica la tasa de suicidio en todos los países y constituye una causa común de muerte en prisión. Tal situación, de máxima gravedad a nivel médico, también puede cuestionar responsabilidades profesionales e institucionales ${ }^{4-6}$. Según un informe del Consejo de Europa, en 2004, la tasa de suicidio en prisión para el conjunto de países de su ámbito fue de $88 / 100.000^{7}$. En las prisiones españolas exceptuando Cataluña, la tasa en el año 2003 fue de 60/100.000 . Entre otros factores, el número de trabajadores dedicados al control y cuidado de los internos podría influir en el número de suicidios consumados en cada prisión ${ }^{9-10}$.

A nivel epidemiológico el suicidio consumado no puede tratarse como una "enfermedad convencional" porque no existe prevalencia, sólo incidencia en poblaciones y circunstancias concretas. Aquí se investiga la incidencia del suicidio en los centros penitenciarios de Cataluña y se comparan los resultados con los publicados para el conjunto de España y para el resto de países europeos. Por otro lado se analizan algunas variables de interés en el momento de establecer planes y protocolos para la prevención del suicidio en los centros penitenciarios. Se ha demostrado que el suicidio se puede prevenir en la medida en que se identifican los factores de riesgo y las variables, intrínsecas y extrínsecas al sujeto, que predisponen y/o facilitan consumar cada acto ${ }^{11-12}$.

\section{MATERIAL Y MÉTODOS}

Se analiza en retrospectiva la frecuencia y características de los fallecimientos por suicidio ocurridos entre 1990 y 2005 entre las personas dependientes del sistema penitenciario catalán. La referencia usada para el cálculo de las tasas de mortalidad fue la población media ingresada en los centros penitenciarios cada año. Los datos para elaborar este estudio proceden del Registro de Mortalidad del Área de Sanidad Penitenciaria, adscrita a la Secretaría General de Servicios Penitenciarios y Rehabilitación de la Generalitat. Estos datos se cruzan periódicamente con los disponibles en el Servicio de Inspección, Régimen y Comisiones Territoriales de Asistencia Social Penitenciaria.

Las variables de estudio fueron: edad, sexo, periodo temporal en que tuvo lugar el suicidio, centro pe- nitenciario, situación familiar, lugar de nacimiento, motivo de ingreso en prisión, tiempo de permanencia en prisión y situación penitenciaria.

En la muestra analizada se calculó la media o promedio ( $\mathrm{m}$ ) como medida de tendencia central, el rango o recorrido (r) como descriptor de dispersión y las tasas y porcentajes como indicadores relativos de frecuencia. Dada la heterogeneidad de los datos bibliográficos no se establecieron pruebas de probabilidad estadística (p), y los resultados más importantes se limitan a cálculos simples. Para discutir los resultados con la situación general en España y en otros países se consideraron fiables los datos publicados por los organismos compententes, así como los aparecidos en la literatura indizada ${ }^{7-16}$. Los datos comparativos fueron tabulados por selección y recopilación.

\section{RESULTADOS}

En la figura 1 se concreta la incidencia anual de suicidio en las prisiones catalanas entre 1990-2005. Durante el período de estudio se registraron 65 muertes por suicidio, lo que representa el $4,7 \%$ del total de población penitenciaria fallecida en el sistema penitenciario catalán. La incidencia anual media de período (15 años) fue de 59/100.000, unas 8 veces por encima de la registrada en la población catalana. El rango en la incidencia anual osciló entre 0 (1992, 1998) y algo por encima de 100 (2002, 2004). Cabe señalar que no todos los fallecimientos fueron dentro de la prisión, 9 tuvieron lugar en el hospital y 4 se encontraban de permiso.

\section{Variables analizadas:}

1) Período temporal en que tuvo lugar el suicidio: Los días con mayor número de suicidios fue-

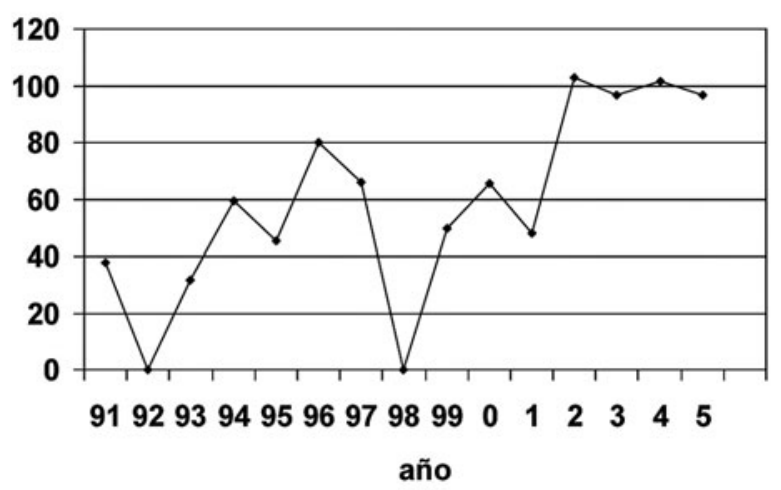

Figura 1. Incidencia de suicido en prisión en Cataluña (1990-2005) (suicidios por 10.000 internos). 
ron los jueves $(\mathrm{n}=13)$ y sábados $(\mathrm{n}=13)$, y con menos, los domingos $(\mathrm{n}=6)$ y viernes $(\mathrm{n}=5)$. El ritmo circanual (figura 2) muestra dos máximos en febrero y septiembre, y un mínimo en enero.

2) Distribución por centros: C.P. Homes Barcelona (33), C.P. Quatre Camins (8), C.P. Can Brians (6), C.P. Ponent (6), otros (12).

3) Situación familiar: solteros (32), casados (15), separados/divorciados (9), con hijos (29), sin hijos (31), no constan hijos (6).

4) Distribución por edades: $77,3 \%$ menos de 40 años, 53,3\% entre 31-40 años.

5) Españoles: $82 \%$, extranjeros: $18 \%$.

6) Tiempo de permanencia en prisión (figura 3 ).

7) Distribución por sexo: en el período estudiado se suicidaron 4 mujeres $(6,8 \%)$.

8) Situación penitenciaria y ubicación: durante el período estudiado el 23,8\% del conjunto de la población reclusa eran preventivos $(r=19,7$ $39,8 \%$ ), mientras que la mayoría de los internos que cometieron el suicidio eran preventivos $(52,5 \%)$. Del total de internos que se suicidaron el $29 \%$ eran primarios, el $26 \%$ se encontraban en el departamento de enfermería y el $14 \%$ en el departamento de ingresos.

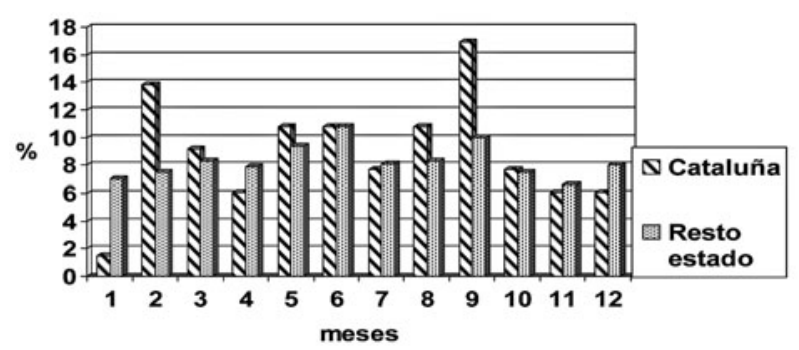

Figura 2. Ritmo circanual del suicidio en las prisiones de Cataluña (1990-2005).

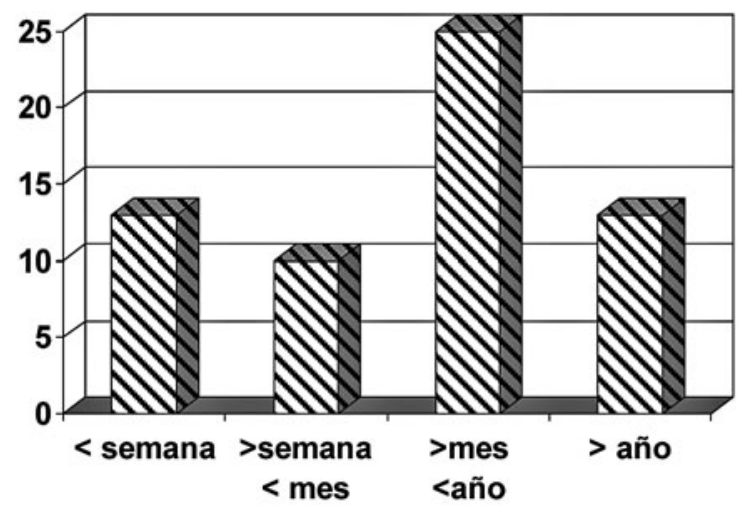

Figura 3. Tiempo de permanencia en prisión de los casos de suicidio en Centros Penitenciarios de Cataluña (1990-2000).

\begin{tabular}{|c|c|c|c|}
\hline PAÍS & En prisión & $\begin{array}{l}\text { En población } \\
\text { general }\end{array}$ & $\begin{array}{l}\text { Razón entre } \\
\text { prisión y } \\
\text { población } \\
\text { general }\end{array}$ \\
\hline Austria & 89 & 27.1 & 2.3 \\
\hline Bélgica & 195 & 31.2 & 6.2 \\
\hline$\overline{\text { Dinamarca }}$ & 168 & 20.2 & 8.3 \\
\hline Finlandia & 87 & 31.9 & 2.7 \\
\hline Francia & 209 & 26.6 & 7.9 \\
\hline Irlanda & 115 & 21.4 & 5.3 \\
\hline Islandia & 0 & 19.6 & 0 \\
\hline Italia & 100 & 11.1 & 9.0 \\
\hline Noruega & 240 & 16.1 & 14.9 \\
\hline Holanda & 71 & 12.7 & 5.5 \\
\hline Portugal & 138 & 18.9 & 7.3 \\
\hline Inglaterra-Galles & 79 & 10.8 & 7.3 \\
\hline Escocia & 120 & & \\
\hline Irlanda del Norte & 253 & & \\
\hline Suiza & 152 & 26.5 & 5.7 \\
\hline
\end{tabular}

Tabla I. Tasa anual de mortalidad por suicidio en prisiones y en población general, expresada en casos por 100.000 (año 2003)

\begin{tabular}{llc}
\hline País & $\begin{array}{c}\text { Proporción } \\
\text { interno/trabajador }\end{array}$ & $\begin{array}{c}\text { Tasa suicidio por } \\
100.000 \text { internos }\end{array}$ \\
\hline Ucrania & 4.4 & 21 \\
\hline Bulgaria & 3.5 & 30 \\
\hline Polonia & 3.3 & 46 \\
\hline Rumanía & 3.2 & 15 \\
\hline Turquía & 2.9 & 53 \\
\hline España & 2.6 & 65 \\
\hline Francia & 2.1 & 53 \\
\hline Alemania & 2.1 & 209 \\
\hline Holanda & 1.6 & 71 \\
\hline Suiza & 1.6 & 152 \\
\hline Escocia & 1.5 & 120 \\
\hline Italia & 1.3 & 100 \\
\hline Finlandia & 1.2 & 87 \\
\hline Suecia & 1.1 & 118 \\
\hline Dinamarca & 1 & 168 \\
\hline Noruega & 1 & 240 \\
\hline Irlanda del Norte & 0.7 & 253 \\
\hline
\end{tabular}

Tabla II. Comparación de proporción interno/trabajador e índice de suicidio por 100.000 reclusos. Datos del Consejo de Europa (año 2003) 
En las tablas I y II se recogen datos orientativos de la incidencia del suicidio en la población general y en el medio penitenciario de los países europeos (2003), así como la relación entre el número de trabajadores/interno en algunos de ellos. En Cataluña, la incidencia del suicidio en prisión en el año 2003 fue de $98 / 100.000$ y en 2004 de $101,5 / 100.000^{13}$, frente al $6,9 \%$ detectado en la población general ese mismo año ${ }^{14}$. En la población penitenciaria del resto de España la incidencia de suicidio en 2003 fue de $65 / 100.000$, ocho veces por encima de la incidencia registrada para la población general española ${ }^{8}$.

\section{DISCUSIÓN}

En Cataluña, la incidencia anual media del suicidio en prisión calculada para un período de 15 años es de 59/100.000, destacando la alta variabilidad interanual de la tasa y el aumento sostenido a partir de 2001, probablemente por el incremento en el número de internos. Esto demuestra la importancia de señalar el período de estudio en el momento de realizar cualquier tipo de comparación. La incidencia del suicidio en Cataluña es similar a la registrada en el resto del territorio nacional tanto en la población general como en la reclusa, coincidiendo en un factor multiplicador de 8 asociado al encarcelamiento.

La incidencia de suicidio en 2003 en los países europeos no parece relacionada con el potencial económico de de cada uno, pero probablemente influyen algunos factores socioculturales. Podrían argumentarse algunas hipótesis en este sentido, que no se analizan aquí, y son motivo de otras investigaciones. $\mathrm{Pa}-$ rece evidente que la diferencia en los tamaños muestrales de la población reclusa en cada país es un factor de confusión muy importante en el momento de comparar la incidencia de suicidio en prisión entre países. Además, la alta variabilidad de la incidencia anual registrada en las prisiones catalanas, debe suponerse también para el resto de países europeos en mayor o menor medida.

En contra de los que cabría esperar el mayor número de trabajadores en el sistema penitenciario no parece relacionarse con la menor incidencia de suicidio (tabla II). En Cataluña, en 2003, la proporción interno trabajador fue de 2,4 y la tasa de suicidos 98/100.000. En el resto de España dicha proporción fue similar, con menos suicidios (65/100.000).

Respecto a la cronobiología del suicidio en prisión nuestros datos coinciden con un estudio francés en el que se observa un incremento los sábados. En cuanto a un posible ritmo circanual se observa un mí- nimo en enero y dos máximos en febrero y septiembre. En el estudio francés, en cambio, se detectaron picos en junio y diciembre. En nuestra población se registró un solo caso en el mes de enero, dato que concuerda con el menor número de suicidios en dicho mes para la población general ${ }^{14}$.

De los internos que se suicidaron 29 tenían hijos frente a 31 que no tenían (no nos consta información de 6 casos). En cuanto a la situación familiar, el colectivo más numeroso (32 casos), era el de los solteros, seguidos de 15 casados y 9 divorciados. En la población general las personas casadas presentan menos riesgo de suicidio que las solteras, divorciadas o viudas.

En cuanto a la edad, el 77,3\% de casos tenía menos de 40 años. La incidencia más alta se presentó en el intervalo de 31 a 40 años (53\% del total de suicidios), lo que sugiere un posible riesgo asociado ya que sólo el 38,2\% de la población reclusa estaba en esa franja.

En cuanto al motivo de estancia en prisión, el $50 \%$ presentaba como delito principal el socioeconómico o contra el patrimonio, el 19,6\% homicidio y el $10,7 \%$ delito contra la libertad sexual. Como referencia comparativa en Cataluña, en el año 2002, el 52\% de internos ingresó por delito contra el patrimonio o socioeconómico, el 6,1\% homicidio y el 5,3\% de tipología sexual.

En la población general la prevalencia del suicidio es menor en el sexo femenino, exceptuando algunas zonas asiáticas. Según datos del Consejo de Europa el porcentaje de mujeres en prisión es de aprox. el $5 \%$. La proporción en Cataluña durante el período estudiado fue del $7 \%$. En nuestra población se suicidaron 4 mujeres, lo que representa un 6,8\% de los suicidios consumados. En cuanto a etnias o país de origen, el $18 \%$ de los casos de suicidio eran extranjeros. No se intuyen diferencias significativas con la población española si se tiene en cuenta que en junio de 1999 el 19,7\% de los reclusos eran extranjeros, alcanzando hasta el 34,2\% en diciembre de 2005.

En relación a la situación penitenciaria cabe destacar que el 52,5\% de los suicidas en la muestra estudiada estaba en situación preventiva, mientras que el $47,5 \%$ eran penados. Estos porcentajes deben compararse con la situación de los presos durante los 15 años estudiados, en la que el $23,8 \%$ de la población reclusa se encontró (de promedio) en situación preventiva, con un rango entre 19,7\%-39,8\%. La situación preventiva parece muy relacionada con el suicidio, en consonancia con la precocidad del mismo tras el ingreso en prisión. Nuestros datos indican que el impacto del ingreso en prisión es un factor de riesgo fundamental de suicidio en la muestra estudiada, puesto que el $21,3 \%$ de los casos se produjeron du- 
rante la primera semana de ingreso en el centro penitenciario. Además el suicidio en el departamento de ingresos está muy por encima del número de internos que permanecen habitualmente en esas dependencias. Esto mismo se ha demostrado estadísticamente en EEUU, Canadá y otros países ${ }^{9,10,18,19}$.

La incidencia del suicidio en las prisiones catalanas se encuentra muy por encima de la población general, como sucede en España y en el resto de países desarrollados, planteando el interrogante de si la prisión constituye un factor de riesgo de suicidio, si las personas con factores de riesgo de suicidio están sobrerepresentadas en la prisión, o si ambas cosas suceden. En Cataluña, como parece ocurrir de modo generalizado, el problema del suicidio en prisión se incrementa a medida que lo hace la población reclusa, por lo que en los últimos años se registra una clara tendencia al alza. La conclusión más importante es la necesidad de prevenir el riesgo de suicidio, muy especialmente durante los primeros días o semanas del ingreso y en sujetos especialmente susceptibles por sus características psíquicas y sociales. Esto justifica la necesidad de que la visita médica en los nuevos ingresos se lleve a cabo lo antes posible al efecto de detectar riesgo suïcida y de aplicar protocolos preventivos que correspondan.

\section{CORRESPONDENCIA}

Talleres Editoriales Cometa, S.A.

Ctra. Castellón, km. 3,400.

50013 Zaragoza.

\section{REFERENCIAS BIBLIOGRÁFICAS}

1. Bertolote JM, Fleischmann A. A global prespective in the epidemiology of suicide. Suicidology 2002; 7: 6-8.

2. Krug EG, Dahlger LL, Mercy JA, Zwi AB, Lozano R. World report on violence and health. Genève, Organisation mondiale de la santé, 2002.

3. Philips Mr, Li Zhang Y. Suicide rates in China 1995-99. Lancet 2002; 359: 835-40.

4. Conacher GN. Management of mentally disordered offenders in prision. Montreal and Kingston: McGill-Queen's University Press, 1993.

5. Barrios Flores LF. El suicidio en Instituciones Penitenciarias I. Responsabilidad institucional. Rev Esp Sanid Penit 2001; 3: 118-127.
6. Barrios Flores LF. El suicidio en Instituciones Penitenciarias II. Responsabilidad profesional. Rev Esp Sanid Penit 2002; 4: 31-38.

7. Council of Europe. Space I. Survey 2004

8. Ministerio del Interior. Dirección General Instituciones Penitenciarias. Subdirección General de Sanidad Penitenciaria. Mortalidad en Instituciones Penitenciarias, 2003.

9. Boureau of Justice Statistics. U.S. Departament of Justice. Office of Justice Programs, 2006.

10. Pratt D, Piper M, Appleby L, Webb R, Shaw J. Suicide in recently released prisoners: a population-based cohort study. L. Lancet 2006; 368: 119-23.

11. Fruehwald S, Frottier P, Ritter K, Eher R, Gutiérrez K. Impact of overcrowding and legislational change on the incidence of suicide in custody: experiences in Austria, 1967-1996. Int J Law Psychiatry 2002; 25: 119 -128.

12. Departament of Health and Human Services. The Surgeon General's call to a to prevent suicide. Washington (DC): Departament of Health and Human Services 1999. Available online from: URL:http://www. surgeongeneral.gov/library/ ca,calltoaction/default.htm.

13. Descriptors estadístics Serveis Penitenciaris i Rehabilitació. Secretaria de Serveis Penitenciaris, Rehabilitació i Justícia Juvenil. Departament de Justícia. Generalitat de Catalunya, 2006.

14. Instituto Nacional de Estadística. http://www. ine.es/inebase/cgi/axi.

15. Preventing Suicide a Resource for prison officers. Mental and Behavioural Disorders. Departament of Mental Health. World Health Organization. Geneve 2000.

16. Zientara-Logeay S. Rapport sur la prevention du suicide en milieu penitentiaire. Sous-Direction de l'Execution des Décisons Judicaires. Bureau de l'Individualisation et des Régimes de Détention. Ministère de la Justice; 1996

17. Centers for Disease Control and Prevention (2004). Suicide Fact Sheet. Available at http:// www.cdc.gov/ncipc/factsheets/suifacts.htm.

18. Moloughney B. Évaluation des besions en soins de santé des détenus sous responsabilité féderérale. Can J Public Health 2004; 95: S1-S69

19. Goos JR, Peterson K, Smith LW, Kalb K, Brodey B. Characteristics of suicide attempts in a large urban jail system with established suicide prevention program. Psychiatr Serv 2002; 53: 574-579. 